\title{
COMMENTARY
}

\section{A balancing act of optimising insulin dose and insulin sensitivity in type 1 diabetes}

\author{
Anna Krook \\ Department of Physiology and Pharmacology, Integrative Physiology, Karolinska Institutet, SE-171 77 Stockholm, Sweden \\ (Correspondence should be addressed to A Krook; Email: anna.krook@ki.se)
}

\begin{abstract}
The incidence and prevalence of type 1, insulin dependent, diabetes is increasing worldwide, spurring efforts to develop and improve therapeutic modalities to improve clinical outcomes for patients. Patients with type 1 diabetes are absolutely dependent on exogenous insulin replacement. Despite advances with novel rapid-acting and intermediateacting insulin analogues, the net result of exogenous delivery
\end{abstract}

is non-physiologic with respect to both timing and the circulating insulin concentrations achieved. This leads to periods of hyperglycaemia and hypoglycaemia, both of which contribute negatively to overall clinical outcome. Thus, better understanding of optimal insulin regimens is of clinical relevance for patients with type 1 diabetes.

Journal of Endocrinology (2011) 211, 1-2
Peripheral insulin resistance is characteristic not only for subjects with type 2 diabetes, but a clinically relevant finding also in subjects with type 1 diabetes (Yki-Järvinen et al. 1987, Rossetti et al. 1990, Bingley et al. 2008). Development of insulin resistance in the context of hypoinsulinaemia and type 1 diabetes has been attributed in part to the hyperglycaemia per se (Yki-Järvinen et al. 1987, Rossetti et al. 1990), although the molecular mechanisms mediating this insulin resistance have not been fully clarified.

In a study published in this issue of Journal of Endocrinology, Okamoto et al. (2011) present evidence that the degree of insulin sensitivity in a rat model of type 1 diabetes presents as a bell-shaped curve in response to different doses of insulin treatment. Rats rendered diabetic were subjected either to saline treatment or to various doses of insulin therapy. The saline-treated rats were markedly insulin resistant, which is in agreement with a hyperglycaemia-induced insulin resistance. Although higher insulin doses, which achieved a near normalisation of glycaemia, resulted in an improvement of insulin sensitivity, these rats were indeed still insulin resistant compared with non-diabetic controls. Interestingly, rats receiving an intermediate insulin dose displayed enhanced insulin sensitivity (even compared with non-diabetic controls) in the face of an only partial normalisation of glycaemia. Insulin-mediated glucose disposal in peripheral tissues is dependent on membrane appearance of the facilitated glucose transporters (GLUTs), in particular, GLUT4. The authors go on to show that the most insulin-sensitive rats (i.e. the rats receiving an intermediate insulin dose) were the ones that displayed the highest increment of insulin-dependent translocation of GLUT4 in white adipose tissue and skeletal muscle.

Diabetes is associated with a reduction in expression of GLUT4 in adipose tissue (Berger et al. 1989). As expected, in the study from Okamoto et al. (2011), diabetes reduced total GLUT4 expression in white adipose tissue and skeletal muscle. In white adipose tissue, insulin therapy increased expression in a dose-dependent manner, with the highest insulin doses resulting in an almost twofold increase in expression over that observed in control rats. In contrast, in skeletal muscle, only the intermediate insulin dose normalised GLUT4 expression to the level in non-diabetic control rats. Thus, increased adipose GLUT4 expression correlates with normalisation of glycaemia, while skeletal muscle GLUT4 expression correlates with insulin sensitivity. The relative impact of disrupted glucose transport in adipose and skeletal muscle has been a long-standing subject of investigation. While skeletal muscle accounts for up to $85 \%$ of glucose disposal following a glucose infusion and around 50\% following glucose ingestion, adipose tissue glucose disposal is more modest. Reduced insulin-mediated glucose uptake into skeletal muscle is noted in subjects with type 2 diabetes as well as in non-diabetic relatives of type 2 diabetic patients. Mice with a targeted deletion of GLUT4 in skeletal muscle 
show fasting hyperglycaemia and are insulin resistant, indicating that a disruption in muscle glucose uptake impacts whole-body glucose metabolism (Zisman et al. 2000). Interestingly, although the overall contribution of adipose to glucose uptake is small, targeted deletion of adipose GLUT4 expression in mice induces insulin resistance (Abel et al. 2001), although this appears to be due in part to a secondary effect on reducing insulin action on other tissues, including skeletal muscle. However, targeted overexpression of GLUT4 at the whole-body level can ameliorate insulin resistance in genetically diabetic $\mathrm{db} / \mathrm{db}$ mice (Gibbs et al. 1995) and adipose-specific overexpression of GLUT4 in transgenic mice leads to enhanced glucose disposal and insulin sensitivity (Shepherd et al. 1993).

The intermediate insulin dose utilised by Okamoto and colleagues also had a significant impact on the liver. Liver expression of PEPCK and G6Pase, as well as liver glycogen was normalised to levels observed in non-diabetic rats. Insulin-stimulation of insulin signalling intermediates was most potent in rats treated with the intermediate insulin dose, with activation of some downstream targets such as IRS2-associated PI 3-kinase and AKT phosphorylation increased above that observed in non-diabetic control rats. Furthermore, nuclear exclusion of FOXO1 was also most efficient in these rats.

The results presented highlight the importance of better understanding of the pathophysiology of insulin resistance and its possible impact on metabolic control also in subjects with type 1 diabetes. Recent investigations indicate that in type 1 patients treated with intensive insulin therapy, insulin resistance is associated with an increased risk of angiopathy and metabolic syndrome, and is a risk factor for cardiovascular morbidity in type 1 diabetes. Understanding the development of insulin resistance in a human context will also be important since there are several indications that the relative contribution of hyperglycaemia versus hyperinsulinaemia may differ with respect to induction of insulin resistance in type 1 diabetes between human and rodents (Rossetti et al. 1990). In human patients, the degree of insulin resistance observed in subjects with type 1 diabetes has been linked to the degree of metabolic control, such that near normal insulin sensitivity has been observed in subjects with well-controlled diabetes (Reveres et al. 1984). Rigorous glycaemic control has also been recommended to reduce microvascular, macrovascular and neuropathic complications for patients with either type 1 or type 2 diabetes. Recent years have seen intensive research into immunomodulatory and $\beta$-cell replacement approaches. Until the time when in vivo glucose sensing and insulin production can be safely restored to type 1 diabetes patients, additional research into developing more sophisticated protocols for exogenous insulin delivery and maintenance of peripheral insulin sensitivity may yield important benefits.

\section{Declaration of interest}

The author declares that there is no conflict of interest that could be perceived as prejudicing the impartiality of the research reported.

\section{Funding}

Research in the author's laboratory is supported by the Swedish Research Council, the European Foundation for the Study of Diabetes, Swedish Diabetes Association, Stockholm County Council, Nova Nordisk Research Foundation, Johan and Jakob Söderbergs Foundation and the diabetes research program at Karolinska Institutet.

\section{References}

Abel ED, Peroni O, Kim JK, Kim Y-B, Boss O, Hadro E, Minnemann T, Shulman GI \& Kahn BB 2001 Adipose-selective targeting of the GLUT4 gene impairs insulin action in muscle and liver. Nature 409 729-733. (doi:10.1038/35055575)

Berger J, Biswas C, Vicario PP, Strout HV, Saperstein R \& Pilch PF 1989 Decreased expression of the insulin-responsive glucose transporter in diabetes and fasting. Nature 340 70-72. (doi:10.1038/340070a0)

Bingley PJ, Mahon JL \& Gale EAM 2008 Insulin resistance and progression to type 1 diabetes in the European Nicotinamide Diabetes Intervention Trial (ENDIT). Diabetes Care 31 146-150. (doi:10.2337/dc07-0103)

Gibbs EM, Stock JL, McCoid SC, Stukenbrok HA, Pessin JE, Stevenson RW, Milici AJ \& McNeish JD 1995 Glycemic improvement in diabetic db/db mice by overexpression of the human insulin-regulatable glucose transporter (GLUT4). Journal of Clinical Investigation 95 1512-1518. (doi:10.1172/JCI117823)

Okamoto M, Anhe G, Silva R, Ferreira M, Freitas H, Mori R, Melo K \& Machado U 2011 Intensive insulin treatment induces insulin resistance in diabetic rats by impairing glucose metabolism-related mechanisms in muscle and liver. Journal of Endocrinology 211 55-64. (doi:10.1530/ JOE-11-0105)

Reveres RR, Kolterman OG, Scarlett JA, Gray RS \& Olefsky JM 1984 Lack of in vivo insulin resistance in controlled insulin-dependent, type I, diabetic patients. Journal of Clinical Endocrinology and Metabolism 58 353-358. (doi:10.1210/jcem-58-2-353)

Rossetti L, Giaccari A \& DeFronzo RA 1990 Glucose toxicity. Diabetes Care 13 610-630. (doi:10.2337/diacare.13.6.610)

Shepherd PR, Gnudi L, Tozzo E, Yang H, Leach F \& Kahn BB 1993 Adipose cell hyperplasia and enhanced glucose disposal in transgenic mice overexpressing GLUT4 selectively in adipose tissue. Journal of Biological Chemistry 268 22243-22246.

Yki-Järvinen H, Helve E \& Koivisto VA 1987 Hyperglycemia decreases glucose uptake in type I diabetes. Diabetes 36 892-896. (doi:10.2337/ diabetes.36.8.892)

Zisman A, Peroni OD, Abel ED, Michael MD, Mauvais-Jarvis F, Lowell BB, Wojtaszewski JFP, Hirshman MF, Virkamaki A, Goodyear LJ et al. 2000 Targeted disruption of the glucose transporter 4 selectively in muscle causes insulin resistance and glucose intolerance. Nature Medicine 6 924-928. (doi:10.1038/78693)

Received in final form 11 July 2011

Accepted 8 August 2011

Made available online as an Accepted Preprint 8 August 2011 